\title{
EXTRACTING DESIGN RECOMMENDATIONS FROM INTERACTIVE GENETIC ALGORITHM EXPERIMENTS: APPLICATION TO THE DESIGN OF SOUNDS FOR ELECTRIC VEHICLES
}

\author{
Souaille, Tom (1); \\ Petiot, Jean-François (1); \\ Lagrange, Mathieu (1); \\ Misdariis, Nicolas (2) \\ 1: Ecole Centrale de Nantes, LS2N; \\ 2: IRCAM
}

\begin{abstract}
The integration of users' perception in the design process is and important challenge for the optimization of products. This study describes how design recommendations can be drawn, from a perceptual experiment with a panel of subjects using a multi-objective interactive genetic algorithm (IGA). The application concerns the bi-objective optimization of the unpleasantness and the detectability of sounds for electric vehicles (EV). After a description of the experimental protocol for the assessment of the detectability and the unpleasantness of EV sounds (listening test), a set of optimal sounds (Pareto efficient) is defined with an IGA experiment. The analysis of these sounds, based on a probabilistic analysis of the selection process, leads to the definition of design recommendations. A second listening test, involving recommended sounds but also other design proposals, allows an evaluation of the validity of the approach. Results show that the sounds recommended obtain interesting performance, in particular to improve the detectability of EV sounds.
\end{abstract}

Keywords: Optimisation, User centred design, Participatory design, sound design, interactive optimisation

\section{Contact:}

Petiot, Jean-François

Ecole Centrale de Nantes

LS2N

France

Jean-Francois.Petiot@ec-nantes.fr

Cite this article: Souaille, T., Petiot, J.-F., Lagrange, M., Misdariis, N. (2021) 'Extracting Design Recommendations from Interactive Genetic Algorithm Experiments: Application to the Design of Sounds for Electric Vehicles', in Proceedings of the International Conference on Engineering Design (ICED21), Gothenburg, Sweden, 16-20 August 2021. DOI:10.1017/pds.2021.418 


\section{INTRODUCTION}

The increasing use of Hybrid or Electric Vehicles (EV) has led to safety concerns for pedestrians (Gillibrand et al., 2011). Below $40 \mathrm{~km} / \mathrm{h}$, the noise emitted by those vehicles is lower than for Internal Combustion Engine Vehicles (ICEV). In urban environments in particular, this makes it potentially more difficult to detect an approaching vehicle. Visually impaired people are particularly affected, as they rely mostly on auditory cues to assess the presence of vehicles (Parizet et al., 2014). Because of this, some countries have started to create a legislation requiring EV to be equipped with a warning sound generation device, as well as specifications regarding the sound that should be emitted (Konet et al., 2011). Several studies have come up with recommendations regarding the nature of such sounds (Misdariis et al., 2013)(Robart et al., 2013)(Poveda-Martinez et al., 2017). These recommendations must also take into account potential noise pollution that could negatively affect the experience of pedestrians, cyclists and other drivers (Petiot et al., 2013).

There is then clearly a conflict between detectability and annoyance for the perception of EV sounds. Different studies addressed this problem (Lee et al., 2017), (Parizet et al., 2014). All these studies are based on hearing tests of a predefined set of sound stimuli.

In a previous paper (Petiot et al., 2019), we proposed a method based on an Interactive Genetic Algorithm (IGA) for the design of EV sounds that takes into account in the same time detectability and unpleasantness. Based on listening tests and a parametric synthesis of EV sounds, it provided efficient solutions, validating the relevance of the approach.

This work is a continuation of this study. It uses the same experimental protocol for the assessment of the detectability and the unpleasantness, but the experiments and the analysis of the results are different. A multiobjective optimization using IGA is now proposed, and design recommendations are drawn from the analysis of the set of Pareto efficient solutions.

The objectives of this paper are:

- To present a method for the analysis of a set of Pareto efficient sounds obtained after a multiobjective IGA experiment, in order to make design recommendations,

- To test the efficiency of recommended designs, compared to other EV sound proposals.

The remainder of the paper is organized as follows. Section 2 presents a background on the experimental protocol for the assessment of detectability and unpleasantness of EV sounds: presentation of the sound synthesis method and description of the scenario for the listening tests. Section 3 presents the material and methods for the experiments and the analysis of the data. A first experiment (experiment 1) uses IGA for a biobjective optimization of the detectability and the unpleasantness, leading to a set of Pareto efficient sounds (termed Optimal_set). The method for the analysis of the optimal set of sounds is presented, in order to draw design recommendations. In a second experiment (experiment 2), design recommendations are assessed and compared to current design proposals. Results are presented in section 4 , and the concluding section provides implications for sound design.

\section{BACKGROUND}

The objective of this section is to make a summary of the EV sounds synthesis method, the scenario for the assessment of the detectability and the unpleasantness, and the IGA implementation.

For more information, the reader is invited to read the initial paper (Petiot et al., 2019).

\subsection{EV sound synthesis}

The EV sounds were synthesized using the mathematical modeling software Matlab and the additive synthesis technique. In order to generate different but plausible sounds for an electric car, after an analysis of current sounds of different carmakers (Misdariis et al., 2012) and personal propositions (Petiot et al, 2013), four main components of the sound were considered.

- Component C1 "A thermic motor sound". This component synthesizes the first harmonics of a classical 4-stroke internal combustion engine (H0.5, H1, H1.5, H2, H4, H6),

- Component C2 “A Harmonic Sound”. This component synthesizes different musical 'notes', harmonic, that constitute a major chord (chord with 3 notes),

- Component C3 and C4: "A broad band Noise". These components synthesize two filtered noises. 
The resulting sound $s(t)$ is finally a weighted sum of these four components (equation 1), to which amplitude modulation is applied, with modulation index $m$ and modulation frequency $f_{m}$ :

$$
s(t)=\left(1+\mathrm{m} \cdot \sin \left(2 \pi f_{m} t\right)\right) \cdot\left(a_{1} \cdot C 1(t)+a_{2} \cdot C 2(t)+a_{3} \cdot C 3(t)+a_{4} \cdot C 4(t)\right)
$$

Since it is out of the scope of this paper to describe all the parameters of the synthesizer (there are more than 70 independent parameters to define a sound), we can mention that all the frequencies and amplitudes of the components are adjustable, to create credible and original sounds. The sound is not constant but controlled by a control parameter of the car: the speed. To make the sound evolve with the speed of the car, we choose to adjust the frequencies and the amplitudes of the different components according to the speed with parameterized patterns.

Among the different synthesis parameters of the sounds, it is necessary to define the optimization variables of the problem, i.e. the variables which are manipulated by the IGA and coded in the genome (design space of the genetic code). After several experiments, the following 6 factors (A, B, C, D, E, F), and their corresponding levels (A1 for level 1 of factor A), were chosen to get a large diversity of sounds (Table 1).

Table 1. Definition of the 6 factors (design variables) and their levels

\begin{tabular}{|c|c|c|c|c|c|}
\hline Factor & Variable & Level 1 & Level 2 & Level 3 & Level 4 \\
\hline $\mathbf{A}$ & $\begin{array}{l}\text { Motor/chord } \\
\text { proportion }\end{array}$ & $a_{2}=0$ & $\frac{a_{1}}{a_{2}}=2$ & $\frac{a_{1}}{a_{2}}=\frac{1}{2}$ & $a_{1}=0$ \\
\hline $\mathbf{B}$ & $\begin{array}{l}\text { Fundamental/center } \\
\text { frequency }\end{array}$ & $\begin{array}{c}f_{1}=f_{3} \\
=80 \mathrm{~Hz} \\
f_{2}=120 \mathrm{~Hz} \\
f_{4}=240 \mathrm{~Hz}\end{array}$ & $\begin{array}{c}f_{1}=f_{3} \\
=120 \mathrm{~Hz} \\
f_{2}=180 \mathrm{~Hz} \\
f_{4}=360 \mathrm{~Hz}\end{array}$ & $\begin{array}{c}f_{1}=f_{3} \\
=160 \mathrm{~Hz} \\
f_{2}=240 \mathrm{~Hz} \\
f_{4}=480 \mathrm{~Hz}\end{array}$ & $\begin{array}{c}f_{1}=f_{3} \\
=200 \mathrm{~Hz} \\
f_{2}=300 \mathrm{~Hz} \\
f_{4}=600 \mathrm{~Hz}\end{array}$ \\
\hline $\mathbf{C}$ & $\begin{array}{l}\text { Harmonic/noise } \\
\text { proportion }\end{array}$ & $a_{1}=a_{2}=0$ & $\frac{a_{3}+a_{4}}{a_{1}+a_{2}}=2$ & $\frac{a_{3}+a_{4}}{a_{1}+a_{2}}=\frac{1}{2}$ & $a_{3}=a_{4}=0$ \\
\hline $\mathbf{D}$ & Number of harmonics & $\begin{array}{l}\text { Motor: } 1 \\
\text { Chord: } 1\end{array}$ & $\begin{array}{l}\text { Motor: } 2 \\
\text { Chord: } 2\end{array}$ & $\begin{array}{l}\text { Motor: } 3 \\
\text { Chord: } 3\end{array}$ & $\begin{array}{l}\text { Motor: } 6 \\
\text { Chord: } 6\end{array}$ \\
\hline $\mathbf{E}$ & $\begin{array}{l}\text { Amplitude } \\
\text { modulation frequency }\end{array}$ & $f_{m}=0.5 \mathrm{~Hz}$ & $f_{m}=2 \mathrm{~Hz}$ & $f_{m}=5 \mathrm{~Hz}$ & $f_{m}=10 \mathrm{~Hz}$ \\
\hline $\mathbf{F}$ & $\begin{array}{l}\text { Amplitude } \\
\text { modulation ratio }\end{array}$ & $\mathrm{m}=0 \%$ & $\mathrm{~m}=17 \%$ & $\mathrm{~m}=33 \%$ & $\mathrm{~m}=50 \%$ \\
\hline
\end{tabular}

With these six factors and four level, the design space counts $4^{6}=4096$ possible designs (all the possible combinations of the full factorial).

\subsection{Listening tests of EV sounds}

\subsubsection{Scenario}

The scenario chosen for the test corresponds to the following situation (Misdariis et al., 2013): a pedestrian located on the sidewalk of a street waits before crossing. An EV may pass by, coming either from the right or from the left. The listener is static, and must indicate when he/she detects the EV.

To obtain a pseudo-realistic passing-by scenario, the following properties have been implemented:

- The sound level of the EV is modulated according to the vehicle/listener distance. The model used, based on acoustic theory, considers the EV as a monopole and provides a sound level inversely proportional to the distance to the listener (1/r) (see Figure 1) (Misdariis et al., 2013), (Lee et al., 2017),

- The speed of the EV is considered as constant and equal to $20 \mathrm{~km} / \mathrm{h}$,

- The duration of the sound stimuli is 15 seconds,

- The Doppler effect (shifting in frequency due to the speed of the source) is taken into account for a more realistic experience,

- The direction of the car (from the right or from the left) is randomly chosen,

- The panning of the EV sound is managed in such a way that the source goes progressively from one canal (left or right, depending of the direction of the EV) to the other (right or left) according to the position of the vehicle. 
Given that the objective of the test is to assess the reaction time associated to the detection of the EV sound, the sound must be incorporated in a background noise (masking signal). The background noise considered in the study corresponds to a stereo street recording of a busy intersection in Paris, France. The level of the background noise was adjusted to a convenient level and kept constant for all the stimuli proposed in the listening test. Figure 1 describes the mixture of the background and the EV sounds and their respective sound level evolution.

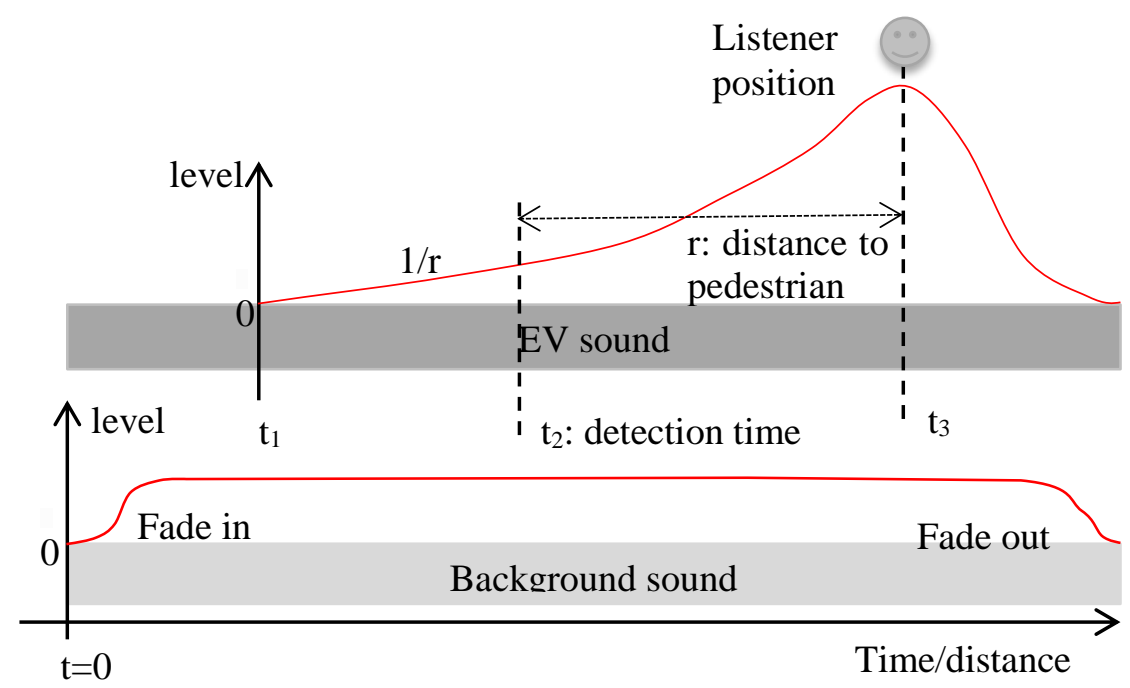

Figure 1. Timeline of the assembly of the background and the EV sound, with their respective level evolution (the $x$ axis represents indifferently the time or the distance of the EV, given that the speed of the vehicle is constant)

\subsubsection{Task of the subject}

The audio stimuli were presented to the participants with a computer interface and Beyerdynamics DT990 headphones in a quiet environment. The same sound output level was set by the experimenter for all computers. Participants had to strike the "space bar" to start playing the stimuli $(t=0)$, and next strike the "a" key as soon as they detect the EV coming from the left, or the "e" key if it is coming from the right (French AZERTY keyboard). This strike allows the definition of the detection time $t_{2}$. To avoid habituation of the participant in the detection time (and detect inconsistent subjects), the starting time $t_{1}$ of the $\mathrm{EV}$ sound in the mixture (figure 2 ) was variable, randomly chosen in the interval $[1,3]$ seconds. The detection duration $D_{d}$ is then given by (equation 2):

$$
D_{d}=t_{2}-t_{1}
$$

After assessing the detectability of the sound, participants were asked to rate the unpleasantness of the sound on a structured semantic scale from " 0 " (not at all unpleasant) to "10" (very unpleasant). These two assessments (detection time, unpleasanteness) constitute the two objectives of the optimization problem that must be minimized.

\subsection{IGA implementation}

The optimization problem addressed in this paper being bi-objective, there are potentially several equally satisfying solutions (Pareto efficient). To address this constraint, the proposed method considers an adaptation of the NSGA-II algorithm (Deb et al., 2002), which aims at finding an approximation of the Pareto front. The solutions are compared based on the so-called crowded-comparison operator:

- A solution is considered better than another one if it has a lower non-domination rank. The non domination rank of a solution corresponds to the non dominated front it belongs to,

- Within a non-dominated front, the solutions are ranked based on their distances to other solutions of the same front in the objective space. Solutions that are further away from other solutions are considered better. This aims at ultimately obtaining solutions that are evenly spread along the optimal Pareto front. In this elitist algorithm, a register of the best solutions evaluated is updated after each generation and is used to create the next one. 
Concerning the operators of the IGA, the procedure was implemented as follows. The first generation of $N_{g}$ solutions was generated using a Latin Hypercube Sample. After evaluation by the user, the best solutions register was initialized with all $N_{g}$ solutions of this first generation. The next generation was created by randomly applying one of the following genetic operators to each solution within the register:

- Mutation: the solution was replicated to the next generation, with one gene value randomly changed,

- Crossover: another solution was selected within the register through a binary tournament based on the crowded-comparison operator. The chromosomes representing the two solutions were combined, in order to create a new one. This was done by randomly selecting a chromosome location, splitting each chromosome into two parts around that location and connecting the first part of one solution with the second part of the other one. The order in which the solutions were combined was chosen randomly,

- Selection: the solution was replicated in the next generation without any modification.

The probability for each operator to be applied is controlled by the crossover rate $\left(c_{r}\right)$, the mutation rate $\left(m_{r}\right)$ and the selection rate $\left(s_{r}\right)$. These values were chosen between 0 and 1 in such a way that $c_{r}+$ $m_{r}+s_{r}=1$. This process was repeated at each generation. A simulation process (Monte-Carlo) was implemented to tune the different parameters of the IGA (see (Poirson et al., 2013) for more information). The size of the register was kept constant, only containing the $N_{g}$ best solutions. At the end of the iterations, the Pareto solutions of a participant $j$ were recorded in a set called Optimal_Set_j.

\section{MATERIAL AND METHODS}

\subsection{Experiment 1: multiobjective optimization with IGA}

32 students (16 males, 16 females) from the École Centrale de Nantes, France, with no reported auditory deficiencies, used the IGA sound optimization tool with the scenario and the sound stimuli described in the "Background" section. They evaluated 11 generations of $N_{g}=9$ sounds, which took approximately half an hour. Values of mutation rate: $m_{r}=0.7$, crossrate: $c_{r}=0.25$, selection rate: $s_{r}=0.05$, were used for the IGA. A high mutation rate was chosen, to preserve diversity in spite of the small number of individuals per generation and to avoid premature convergence.

The union for all the participants of all the Pareto solutions that satisfy the safety criterion was formed.

$$
\text { Optimal_set }=\bigcup_{\mathrm{j}=1}^{\mathrm{m}}\left(\text { Optimal_set }_{j}\right)
$$

Optimal_Set represents a selection of EV sounds that, from a perceptual point view, make a satisfying trade-off between detectability and unpleasantness. To provide information that could be used as recommendations for a sound designer, an analysis of these sounds and the most occurring factors levels in Optimal_Set is conducted.

\subsection{Analysis of the sound of Optimal_set}

The principle of the method to draw design recommendations from the analysis of the designs in Optimal_Set is to consider the selection process of the designs made by the participants as a random process that depends on probability laws. The set Optimal_set, of size N, is a subset of the sample space $\Omega$ (full factorial design). From the chosen designs in Optimal_Set, estimates of the parameters of these probability laws can be calculated. And with these parameters, it becomes possible to make inference and provide a probability score for any design of the design space.

\subsubsection{Joint probability}

Given the sample space $\Omega$ (set of all possible designs of the design space), and the design variables $\mathrm{X}_{i},(i=1$ to 6$)$ that describe the design, the first model that can be made is to assume that the choice of the designs in Optimal_set depends on all the variables and all their possible interactions.

In this case, the probability law of the selection process of any design $d$ defined by the design variables $\mathrm{X}_{i},(i=1$ to 6$), \mathrm{d}=\left(X_{1}=x_{1}, X_{2}=x_{2}, \ldots, X_{6}=x_{6}\right)$ by the IGA experiments is given by the joint probability:

$$
\mathrm{P}(\mathrm{D}=\mathrm{d})=\mathrm{P}\left(X_{1}=x_{1}, X_{2}=x_{2}, \ldots, X_{6}=x_{6}\right)=\frac{\text { card }\{\text { D } \in \text { optimal_set } / D=d\}}{\text { card }(\text { optimal_set })}
$$


Where card represents the cardinality of a set (number of elements)

For example, if a design is present once in Optimal_set, its probability is $\mathrm{P}=\frac{1}{\operatorname{card}(\text { optimal_set })}$, if it is not chosen, its probability is $\mathrm{P}=0$.

This 6-dimensions joint probability is not so interesting to make design recommendations because it is only able to recommend designs that are present (and abundant) in Optimal_set. To be able to make recommendations on the levels of the design variables $\mathrm{X}_{i}$, it is necessary to make assumptions on the independence of the variables in the selection process.

\subsubsection{Marginal probability}

If we consider that the variables $X_{i},(i=1$ to 6$)$ are mutually independent in the selection process (no interaction between them), then the probability law of the selection process of any design $\mathrm{d}=\left(X_{1}=\right.$ $\left.x_{1}, X_{2}=x_{2}, \ldots, X_{6}=x_{6}\right)$ is given by:

$$
\mathrm{P}\left(X_{1}=x_{1}, X_{2}=x_{2}, \ldots, X_{6}=x_{6}\right)=\prod_{i=1}^{6} \mathrm{P}\left(X_{\mathrm{i}}=x_{\mathrm{i}}\right)
$$

This is simply the product of the marginal probabilities, where:

$$
\mathrm{P}\left(X_{\mathrm{i}}=x_{\mathrm{i}}\right)=\frac{\text { card }\left\{\text { D } \in \text { optimal_set } / X_{i}=x_{i}\right\}}{\text { card(optimal_set })}
$$

In this case, the designs recommended by the model (designs with the largest probability), is the designs with the most occurring level (largest size) for each variable. Of course the mutual independence of all the variables is a very strong assumptions that only holds if there is no interaction between the variables in the selection process (in the perception of participants). This is rather unlikely in design where the global assessment of a product may be different to the sum of the assessments of each of its variables (Sylcott et al., 2015).

\subsubsection{Independence checking of the variables}

The two previous models being not satisfactory to make design recommendations, it is interesting to propose a model that is based on assumptions that can be checked concerning the independence of the variables in the selection process. That's why we propose first to check the independences between the variables, from the choices made in Optimal_set.

Our proposal is to check, with a statistical test, the independence of any pairs of variables in Optimal_set. We propose to use the chi-square independence test to determine whether there is a significant association between two qualitative variables.

For example, suppose that the pairwise independence test shows that the two groups of variables $\left\{X_{1}, X_{2}, X_{3}\right\}$ and $\left\{X_{4}, X_{5}, X_{6}\right\}$ are independent. This can be shown in the pairwise comparison matrix (see example in Table 3 ).

Then it is possible to use a model where the probability law of the selection process of any design $\mathrm{d}=$ $\left(X_{1}=x_{1}, X_{2}=x_{2}, \ldots, X_{n}=x_{n}\right)$ is given by:

$$
\mathrm{P}\left(X_{1}=x_{1}, X_{2}=x_{2}, \ldots, X_{6}=x_{6}\right)=\mathrm{P}\left(X_{1}=x_{1}, X_{2}=x_{2}, X_{3}=x_{3}\right) . \mathrm{P}\left(X_{4}=x_{4}, X_{5}=x_{5}, X_{6}=x_{6}\right)
$$

Where:

$$
\begin{aligned}
& \mathrm{P}\left(X_{1}=x_{1}, X_{2}=x_{2}, X_{3}=x_{3}\right)=\frac{\text { card }\left\{D \in \text { optimal_set } / X_{1}=x_{1}, X_{2}=x_{2}, X_{3}=x_{3}\right\}}{\text { card }(\text { optimal_set })} \\
& \mathrm{P}\left(X_{4}=x_{4}, X_{5}=x_{5}, X_{6}=x_{6}\right)=\frac{\text { card }\left\{D \in o p t i m a l \_s e t / X_{4}=x_{4}, X_{5}=x_{5}, X_{6}=x_{6}\right\}}{\text { card }(\text { optimal_set })}
\end{aligned}
$$

It is then possible to calculate the probabilities of all the designs $\mathrm{d}=\left(X_{1}=x_{1}, X_{2}=x_{2}, \ldots, X_{6}=\right.$ $x_{6}$ ) of the design space. Designs with the largest probabilities should be recommended.

Some of them are of course present in Optimal_set, but it is likely that designs not present in Optimal_set get a high probability, and be interesting for the design problem.

The method presented allows uncovering combinations of the levels of the variables that are efficient for the objectives considered in the study. It is the strong point of the method: to make recommendations according to the values of the variables instead of the designs themselves. This information is of course more interesting for designers that may take into account recommendations, but also be free to adjust the design according to personal orientations and other constraints or objectives, not taken into account in the objective functions optimized. 


\subsection{Experiment 2: validation test}

After the analysis of Experiment 1, a second panel of subjects (different of the previous panel) was asked to assess the detectability and unpleasantness of different EV sound proposals, including recommended designs with the previous method. 17 students (14 males, 3 females) from the École Centrale de Nantes, France, with no reported auditory deficiencies, participated to the test with the protocol described in the "Background" section. $18 \mathrm{EV}$ sounds were proposed to the evaluation:

- 8 sounds ( $r a n d l$ to rand8 - category random) randomly defined in the experimental space,

- 2 sounds (design1 and design2 - category designed), designed by a sound designer with instructions for "good detectability" and "low unpleasantness",

- 8 sounds (recol to reco8 - category reco), recommended by the method described in the previous sections (largest probability).

To be able to assess the experimental error in the assessments, 4 repetitions of each sound were proposed. In total, each participant had to assess $72(18 * 4)$ EV sounds, proposed in a random order. The global performances of the sounds and of the sound categories (random, designed, reco) were compared using Duncan Multiple Comparison tests.

\section{RESULTS}

\subsection{Experiment 1: IGA test}

To show the convergence of the solutions across the different generations, the fitness (N.B. the lower, the better) of the entire population of sounds can be examined. Since there are two objectives, the fitness can be reduced to the sum of the two objectives (the value of the detection time has been scaled so that its range matches the one of the unpleasantness). Of course, this is not how the IGA operates (it is a multiobjective optimization) but this reduction to a single objective is a convenient way to display the results. Figure 2 (left) shows the average sum of the two objectives calculated across all the generations, and averaged over all participants, with the standard error bars. This is plotted for two conditions, the mean value of the solutions of a generation, and the minimum value of a generation. A diminution of the fitness is noticed, both for the mean and the minimum value. This means that the adaptation of the EV sounds generated by the IGA to the problem is noticeable after 6 or 7 generations. This is also a sign of the reliability of the experimental protocol for the assessment of the detection time and the unpleasantness, and a correct tuning of the IGA parameters.
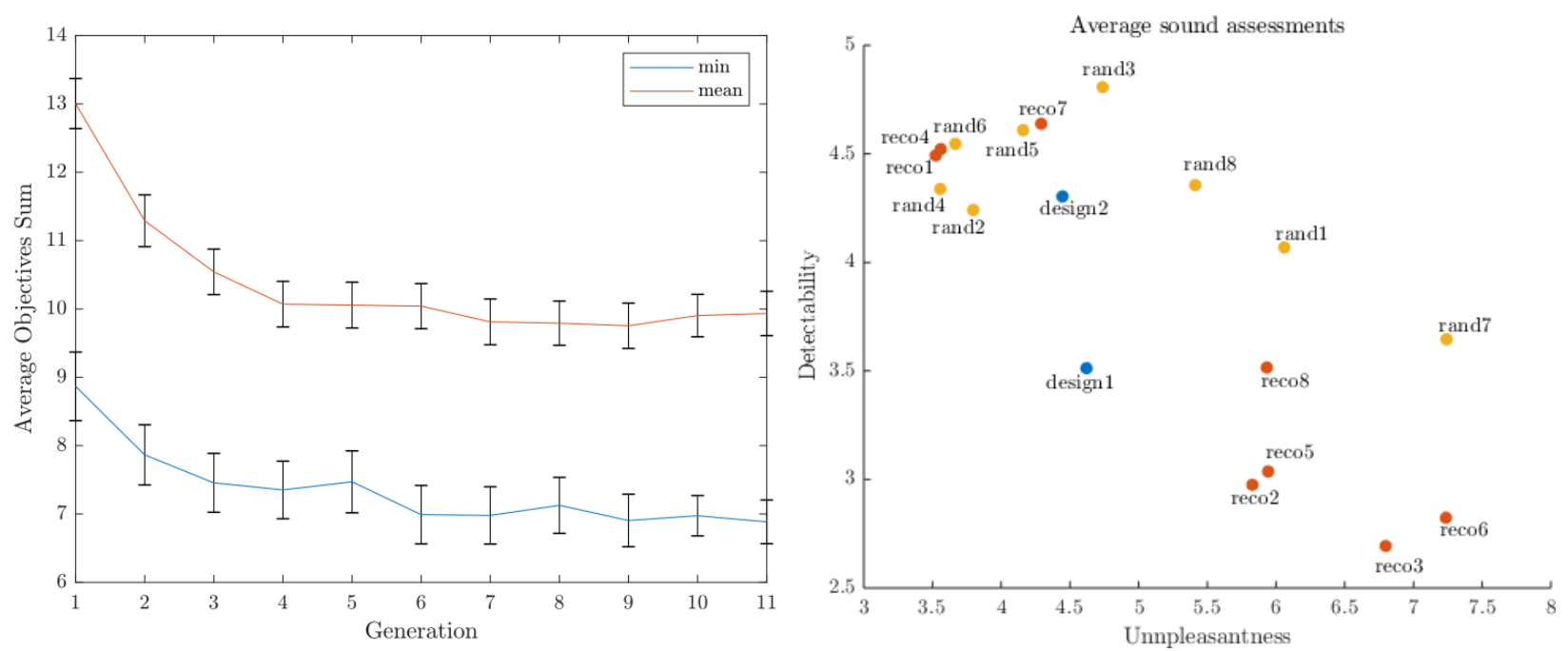

Figure 2. (left): Average values of the sum of the two objectives (unpleasantness and detectability) by generation. (right): Scatterplot of the average performances of the different EV sounds

The Optimal_Set counts N=113 sounds. All designs are present once, except d1(A1 B4 C4 D4 E2 F1) present twice and d2(A2 B4 C4 D4 E1 F1) present 4 times. The occurrences of each level of the variables in Optimal_Set are given in Table 2. To define the variables for which the occurrence of the levels is not equiprobable, a multinomial goodness of fit test of the distribution of the occurrences was carried out. 
Table 2. Occurrences of the levels of the variables in Optimal_Set

\begin{tabular}{|l|l|l|l|l|l|l|}
\hline & A & B & C & D & E & F \\
\hline Level 1 & 35 & $\mathbf{1 4}$ & 19 & 27 & 39 & 36 \\
\hline Level 2 & 20 & 36 & 21 & 28 & 23 & 33 \\
\hline Level 3 & 31 & 33 & 23 & 25 & 28 & 24 \\
\hline Level 4 & 27 & 30 & $\mathbf{5 0}$ & 33 & 23 & 20 \\
\hline $\begin{array}{l}\text { Multinomial } \\
\text { test Signif. }\end{array}$ & N.S. & $*$ & $* *$ & N.S. & N.S. & N.S. \\
\hline & $*: \mathrm{p}<0.05$ & $* *$ p $<0.01$ & N.S.: not significant \\
\hline
\end{tabular}

Only two variables, $\mathbf{B}$ (frequency) and $\mathbf{C}($ Harmonic/noise proportion), obtain occurrences significantly different from a random distribution at the 5\% level. For the frequency of the sounds, the level B1 (low frequency) is under-represented (size $=14$ ). For the Harmonic/noise proportion, the level C4 (broad band noise absent) is over-represented (size $=50$ ). For the whole group of participants, it seems necessary to avoid low frequencies and broadband noise for the high detectability and low unpleasantness of EV sounds. For the other variables (and their possible interactions), it is not possible to make recommendations with this simple sorting one variable at once.

\subsection{Analysis of the sound of Optimal_set}

\subsubsection{Independence test of the variables}

With the definition of the $\mathrm{N}$ designs of Optimal_set, contingency tables of all pairs of variables were formed. The results of the Chi-square test of independence (p-value) are given in Table 3.

The p-values corresponding to a rejection of the independence are presented in bold (a Bonferroni correction was applied to deal with the multiple comparisons problem - threshold value of $0.05 / 15=$ $0.003-15$ is the number of pairs). For the non-significant pairs, test shows that the dependence in the sample is too weak to distinguish it from independence. From this table, the corresponding dependence graph can be drawn (Table 3).

Table 3. Pairwise comparison matrix of the Chi-square test of independence ( $p$-value)

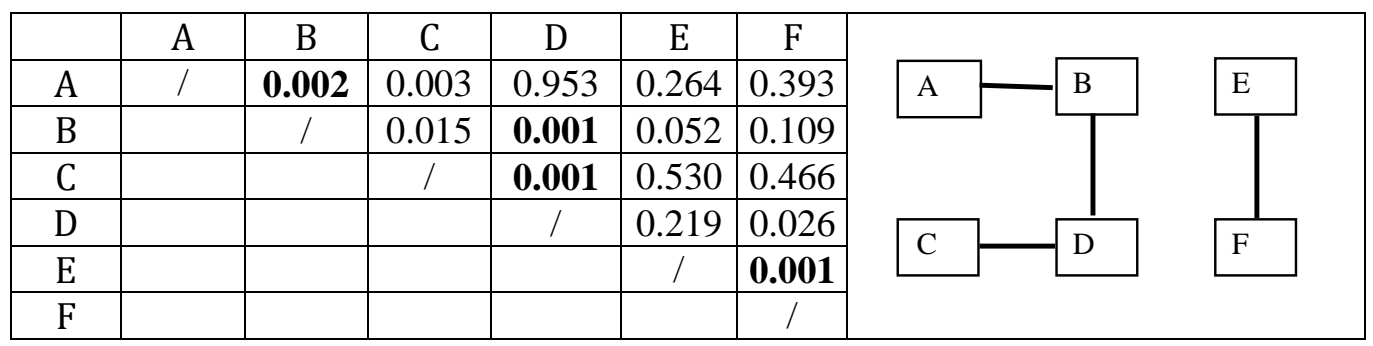

The graph shows that two groups of independent variables can be considered in Optimal_set: $\{A, B, C, D\}$ and $\{E, F\}$. The probability law of the selection process is then given by:

$$
\begin{gathered}
\mathrm{P}\left(\mathrm{A}=A_{i}, \mathrm{~B}=B_{j}, \mathrm{C}=C_{k}, \mathrm{D}=D_{l}, \mathrm{E}=E_{m}, \mathrm{~F}=F_{n}\right)=\mathrm{P}\left(\mathrm{A}=A_{i}, \mathrm{~B}=B_{j}, \mathrm{C}=C_{k}, \mathrm{D}=D_{l}\right) \cdot \mathrm{P}(\mathrm{E}= \\
\left.E_{m}, \mathrm{~F}=F_{n}\right)
\end{gathered}
$$

The probability can be calculated for all the design samples. Designs with the largest probability are recommended.

\subsubsection{Design recommendations}

The 8 designs with the largest probability are presented in Table 4 . They present combinations of variables that are interesting for the optimization problem.

\subsection{Experiment 2: validation test}

\subsubsection{Multiple comparisons}

From the assessments of the participants according to detectability and unpleasantness, the average scores of detectability and unpleasantness of the $18 \mathrm{EV}$ sounds was calculated. To investigate the 
differences in the performances of the sounds according to their category (reco, designed, random), the average scores with their standard errors are presented in Figure 3 left (Unpleasantness) and right (Detection time). To study the differences in the average score, a Duncan multiple comparison test is carried out for every pair of sounds (significant threshold: $\mathrm{p}=0.05$ ). The results are presented with bold lines connecting the sounds in the figures. When sounds are connected, pairs are not significantly different, whereas they are when not connected.

Table 4. Definition of the 8 recommended designs (design variables' levels)

\begin{tabular}{|c|c|c|c|c|c|c|}
\hline & A & B & C & D & E & F \\
\hline reco1 & $\mathrm{A} 3$ & $\mathrm{~B} 2$ & $\mathrm{C} 2$ & $\mathrm{D} 2$ & $\mathrm{E} 1$ & $\mathrm{~F} 1$ \\
\hline reco2 & $\mathrm{A} 1$ & $\mathrm{~B} 4$ & $\mathrm{C} 4$ & $\mathrm{D} 4$ & $\mathrm{E} 1$ & $\mathrm{~F} 1$ \\
\hline reco3 & $\mathrm{A} 2$ & $\mathrm{~B} 4$ & $\mathrm{C} 4$ & $\mathrm{D} 4$ & $\mathrm{E} 1$ & $\mathrm{~F} 1$ \\
\hline$r e c o 4$ & $\mathrm{~A} 3$ & $\mathrm{~B} 2$ & $\mathrm{C} 2$ & $\mathrm{D} 2$ & $\mathrm{E} 3$ & $\mathrm{~F} 2$ \\
\hline reco5 & $\mathrm{A} 1$ & $\mathrm{~B} 4$ & $\mathrm{C} 4$ & $\mathrm{D} 4$ & $\mathrm{E} 3$ & $\mathrm{~F} 2$ \\
\hline reco6 & $\mathrm{A} 2$ & $\mathrm{~B} 4$ & $\mathrm{C} 4$ & $\mathrm{D} 4$ & $\mathrm{E} 3$ & $\mathrm{~F} 2$ \\
\hline reco7 & $\mathrm{A} 1$ & $\mathrm{~B} 2$ & $\mathrm{C} 4$ & $\mathrm{D} 2$ & $\mathrm{E} 1$ & $\mathrm{~F} 1$ \\
\hline reco8 & $\mathrm{A} 4$ & $\mathrm{~B} 3$ & $\mathrm{C} 4$ & $\mathrm{D} 2$ & $\mathrm{E} 1$ & $\mathrm{~F} 1$ \\
\hline
\end{tabular}

According to unpleasantness, the recommended designs (reco) are significantly more unpleasant than the two other categories (Figure 3 left).

According to detection time, all the categories are significantly different: the recommended designs (reco) are significantly more detectable than the two other categories (Figure 3 right).

The results are a little disappointing because they don't show that in average, the recommended designs are always better than the other proposals. But given that the objective are conflicting, we cannot rule out the assumptions that it is even not possible to find, in our design space, 8 designs that dominates all the designs of our design space.
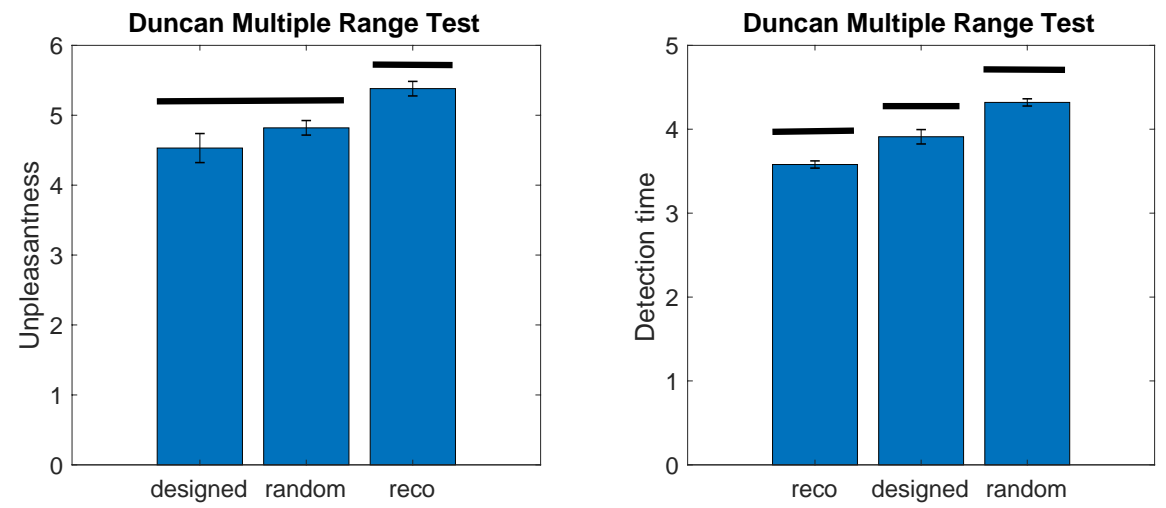

Figure 3. Bar graph of the average value of the unpleasantness (left) and detection time (right) for the different EV sounds categories. Non significant differences between pairs of sounds ( $p>.05)$ are linked with an horizontal line (Duncan multiple comparisons test).

The method presented is still interesting because it allows the definition of EV sounds that are significantly more detectable than other proposals.

\subsubsection{Average assessments}

To have a more accurate view of the results, Figure 2 (right) presents the average performances of each $\mathrm{EV}$ sounds of the three categories (reco, designed, random) according to detection time and unpleasantness.

It is clear on Figure 2 that all the recommended sounds (reco) do not Pareto-dominate all the other sounds. The Pareto front is made of 3 recommended sounds (reco3, reco2, recol), one designed sound (Design1) and 2 random sounds (rand 2 and rand4).

It is noticeable that the range of the random sounds according to unpleasantness is large, but very narrow for detectability: it is unlikely to obtain detectable EV sounds by choosing them randomly.

The two designed sounds obtain average performances, the sounds Designl (Pareto efficient) being an interesting trade-off between the two objectives. 
It is also noticeable on Figure 2 (right) that the ranges of the reco sounds according to unpleasantness and detectability are large. The most detectable EV sounds are recommended sounds (reco2, reco5, reco3 reco6).

Sounds that are highly detectable seem to be very particular, given that neither random nor designed sound obtains comparable performances. The main result is that the recommendation method and the experimental protocol using the IGA allow the determination of very detectable EV sounds. The price to pay is an increase of the unpleasantness. The recommendation method presented in this paper produces interesting results to help the work of a sound designer.

\section{CONCLUSION}

This paper presented a method to make design recommendations after an experiment with an interactive genetic algorithm (IGA). The application concerns the bi-objective optimization of the unpleasantness and the detectability of sounds for Electric Vehicles, and uses listening tests with a panel of participants and an IGA for the multi-objective optimization.

Based on an analysis of the sounds of the Pareto front of all the participants, the method to make recommendations studies the independence of the design variables and calculates a probability score of the designs, in order to recommend the designs with the largest probabilities. A validation experiment shows that the results are promising: even if the recommended designs do not over-rank proposals of a designer according to both objectives, they obtain very good performances according to detectability. The next stage of the project will be to explain the relationships between the design variables of the sounds and the two objectives.

\section{REFERENCES}

Deb, K., Pratap, A., Agarwal, S., and Meyarivan, T. (2002). A fast and elitist multiobjective genetic algorithm: NSGA-II. IEEE Transactions on Evolutionary Computation, 6(2), 182-197.

Gillibrand, A., Suffield, I., Vinamata, X., Williams, R. and Brückmann, A. (2011), “An Initial Study to Develop Appropriate Warning Sound for a Luxury Vehicle Using an Exterior Sound Simulator". SAE International, 2011-01-1727.

Konet, H., Sato, M., Schiller, T., Christensen, A., Tabata, T., and Kanuma, T., (2011), "Development of Approaching Vehicle Sound for Pedestrians (VSP) for Quiet Electric Vehicles", SAE International, 201101-0928.

Lee S.K., Lee S.M., Shin T. and Han M. (2017), “Objective evaluation of the sound quality of the warning sound of electric vehicles with a consideration of the masking effect: annoyance and detectability". International Journal of Automotive Technology, 18(4), pp. 699-705 (2017). https://dx.doi.org/10.1007/s12239-017-0069-6.

Misdariis, N., Cera, A., Levallois, E., and Locqueteau, C., (2012), "Do electric cars have to make noise? An emblematic opportunity for designing sounds and soundscapes". Proc. of Acoustics 2012, Nantes, France.

Misdariis N., Gruson A. and Susini P. (2013), "Detectability study of warning signals in urban background noises: A first step for designing the sound of electric vehicles”. Proc. Mtgs. Acoust. 19, 040032 (2013); https://dx.doi.org/10.1121/1.4799454.

Parizet E., Ellermeier W. and Robart R. (2014), “Auditory warnings for electric vehicles: Detectability in normal-vision and visually-impaired listeners”, Applied Acoustics, Volume 86, Pages 50-58.

Petiot, J-F., Legeay, K., and Lagrange, M. (2019). Optimization of the Sound of Electric Vehicles According to Unpleasantness and Detectability. Proceedings of the Design Society: International Conference on Engineering Design, 1(1), 3949-3958. https://dx.doi.org/10.1017/dsi.2019.402

Petiot J-F., Kristensen B.G. and Maier A.M. (2013), "How should an electric vehicle sound? User and expert perception". Proceedings of IDETC/CIE 2013, August 4-7, 2013, Portland, Oregon, USA.

Poirson E., Petiot J-F., Boivin L. and Blumenthal D. (2013), "Eliciting User Perceptions Using Assessment Tests Based on an Interactive Genetic Algorithm”. ASME. J. Mech. Des. 135(3):031004-031004-16.

Poveda-Martínez, P., Peral-Orts, R., Campillo-Davo, N., Nescolarde-Selva, J., Lloret-Climent, M., and RamisSoriano, J. (2017). Study of the effectiveness of electric vehicle warning sounds depending on the urban environment. Applied Acoustics, 116, 317-328.

Robart, R., Parizet, E., Chamard, J. C., Janssens, K. and Biancardi, F. (2013), “eVADER: A perceptual approach to finding minimum warning sound requirements for quiet cars". AIA-DAGA 2013 Conf. Acoustics, 1.

Sylcott B., Michalek J. J. and Cagan J. 2015. Exploring the role of interaction effects in visual conjoint analysis. Journal of Mechanical Design, Vol. 137. 